\title{
Lymphocyte labile iron pool, plasma iron, transferrin saturation and ferritin levels in colon cancer patients ${ }^{*}$
}

\author{
Daniel Gackowski ${ }^{1}$, Marcin Kruszewski ${ }^{3,4}$, Zbigniew Banaszkiewicz ${ }^{2}$, \\ Arkadiusz Jawien ${ }^{2}$ and Ryszard Olinski ${ }^{1}$ 凶 \\ ${ }^{1}$ Department of Clinical Biochemistry, ${ }^{2}$ Department and Clinic of Surgery, Ludwik Rydygier \\ Medical University in Bydgoszcz, Bydgoszcz, Poland; ${ }^{3}$ Institute of Nuclear Chemistry and \\ Technology, Department of Radiobiology and Health Protection, Warszawa, Poland; ${ }^{4} M$. \\ Sklodowska-Curie Memorial Cancer Center and Institute of Oncology, Department of \\ Experimental Hematology and Cord Blood Bank, Warszawa, Poland
}

Received: 23 October, 2001; revised: 10 January, 2002; accepted: 27 February, 2002

Key words: colon cancer, iron metabolism, labile iron pool

\begin{abstract}
Patients with colorectal carcinoma showed statistically significant lower values of transferrin saturation, total iron binding capacity and serum iron level as compared with control group, while the level of ferritin and the size of labile iron pool in carcinoma patients were higher, although this difference was not statistically significant. Our observations are in favour of the hypothesis which suggests that changes in iron metabolism restrict iron availability for tumour cells and as consequence, slow their growth.
\end{abstract}

Iron is an essential component in the cellular metabolism. It is a component of many enzymes and other proteins. Iron metabolism in organisms is well controlled by binding to proteins such as transferrin and ferritin to minimize the amount of "free iron pool" or "labile iron pool”- LIP (low molecular mass iron). The presence of free iron in a cell can result in the production of reactive oxygen species such as hydroxyl radical, which can damage biomolecules including DNA and lipids [1]. Also epidemiological data suggest that iron metabolism may play a role in the development of cancer [2, 3].

In the case of colorectal cancer and colon adenoma in humans, opinions on the associa-

\footnotetext{
This work was financed by grant from the State Committee for Scientific Research (No. 4P05D03017) and the Maria Sklodowska-Curie Polish-American Joint Fund II (No. MZ/NIST-97-298).

${ }^{\square}$ Address for correspondence: Prof. Ryszard Olinski, Department of Clinical Biochemistry, Ludwik Rydygier Medical University in Bydgoszcz, M. Karłowicza 24, 85-092 Bydgoszcz, Poland; tel. (48 52) 585 3744; fax. (48 52) 585 3771; e-mail: ryszardo@aci.amb.bydgoszcz.pl
}

Abbreviations: $\mathrm{Me}_{2} \mathrm{SO}$, dimethylsulfoxide; LIP, labile iron pool. 
tion with iron metabolism are varied [3, 4]. Therefore in the present study we examined a broad spectrum of iron metabolism parameters in the blood of colorectal carcinoma patients. Additionally determination of vitamin $\mathrm{C}$, which may influence iron intake was also included. Since LIP can influence the severity of oxidative stress and this in turn may contribute to lymphocyte dysfunction, i.e. to the immunological response in cancer patients [5], our attention was focused on LIP content in lymphocytes of colon cancer patients as compared to healthy subjects.

\section{MATERIALS AND METHODS}

Patients. The study was conducted in two groups of subjects. The control group consisted of 51 healthy males $(n=21)$ and females $(\mathrm{n}=30)$ with a mean age 60 years (range 26 to 87 years). The colorectal cancer patients group of 45 comprised 26 males and 19 females. All the patients had histologically proven adenocarcinomas with $\mathrm{G} 2(\mathrm{n}=35)$ and G3 $(n=16)$ staging. No differences in the investigated parameters were found between the staging groups. The mean patient age was 65 years (range 44 to 90 ). None of the subjects from either groups were smokers.

The study was approved by the medical ethics committee of The L. Rydygier Medical University, Bydgoszcz, Poland, No. 10/2000 (in accordance with Good Clinical Practice, Warsaw 1998) and all the patients gave informed consent.

Determination of plasma vitamin $C$ (ascorbic acid) by HPLC. Quantification of vitamin $\mathrm{C}$ by HPLC was as described previously [6].

Isolation of lymphocytes from venous blood. Peripheral blood was collected by vein puncture. Each blood sample was diluted with RPMI medium (Sigma) and divided into two aliquots. The blood was carefully applied on top of Histopaque 1077 solution (Sigma) and lymphocytes were isolated by centrifugation according to the procedure recommended by the manufacturer. For further use the isolated lymphocytes were slowly frozen $\left(1^{\circ} \mathrm{C} / \mathrm{h}\right)$ in a bovine serum solution containing $20 \%$ $\mathrm{Me}_{2} \mathrm{SO}$.

Determination of labile iron pool. To measure the intracellular labile iron pool (LIP) in human lymphocytes in situ we used the fluorescent assay recently developed by Epsztejn et al. [7]. This assay is based on the quenching of the fluorescent calcein by metal ions. In brief, living cells in suspension are loaded with a cleavable, permeant calcein acetoxymethyl ester and left for at least $20 \mathrm{~min}$ at $37^{\circ} \mathrm{C}$. Baseline fluorescence of the cell suspension is then recorded. After a stable baseline is attained, a highly permeant iron chelator is added to the loaded cells. The dequenching of the fluorescence of calcein-iron complex is recorded. The extent of fluorescence dequenching evoked by the added chelator provides the measure of intracellular labile iron pool. The intracellular concentration of calcein is determined by titration of the cell suspension with free calcein standards [8].

Determination of ferritin, transferrin saturation and total iron in blood serum. Ferritin, transferrin saturation and iron were analysed using Vidas and Olympus AutoAnalyzers during routine laboratory tests.

\section{RESULTS AND DISCUSSION}

Body iron stores and dietary iron intake have been shown to be positively correlated with the risk of colon cancer [3, 9]. Surprisingly, in our study carcinoma patients showed statistically significant lower values of transferrin saturation, total iron binding capacity and serum iron level when compared with a control group (Table 1). There may be several reasons which can explain these discrepancies: (i) in our work patients with fully developed colon cancer were studied while in the aforementioned papers body iron stores were investigated in apparently healthy 
groups with only the documented possible risk of cancer development was discussed. [2, 10]; (ii) ascorbate could favour an excessive uptake of iron [11, 12]. Since the concentration of vitamin $\mathrm{C}$ was lower in the blood of cancer patients than in healthy subjects this may contribute to the decreased concentration of body iron stores (Table 1); (iii) another cause
Mean values of the "labile iron pool" in lymphocytes of the patient group were higher than in the control group although these differences were not statistically significant. The lack of significant differences in LIP and ferritin concentrations, observed in this study, may be explained, at least in part, by the large individual variability of these param-

\section{Table 1. Analytical data.}

The presented data are means of 51 and 45 subjects in the control and patients groups, respectively. Labile iron was determined in 29 and 25 subjects, respectively.

\begin{tabular}{lccc}
\hline & $\begin{array}{c}\text { Control group } \\
\text { (mean } \pm \text { S.D.) }\end{array}$ & $\begin{array}{c}\text { Patients } \\
\text { (mean } \pm \text { S.D.) }\end{array}$ & p-value \\
\hline Total plasma iron concentration $(\mu \mathrm{g} / \mathrm{dl})$ & $106.92 \pm 36.64$ & $63.00 \pm 50.28$ & $0.0000035^{*}$ \\
Total iron binding capacity $(\mu \mathrm{g} / \mathrm{dl})$ & $355.17 \pm 65.09$ & $283.60 \pm 97.42$ & $0.000045^{*}$ \\
Plasma transferrin saturation $(\%)$ & $29.72 \pm 8.56$ & $22.49 \pm 15.81$ & $0.0056^{*}$ \\
Plasma ferritin concentration $(\mathrm{ng} / \mathrm{ml})$ & $204.93 \pm 207.21$ & $232.31 \pm 278.09$ & 0.5830 \\
LIP in lymphocytes $(\mu \mathrm{M})$ & $0.5292 \pm 0.5773$ & $0.6968 \pm 0.8138$ & 0.3820 \\
Plasma ascorbic acid concentration $(\mu \mathrm{M})$ & $52.05 \pm 29.04$ & $30.66 \pm 28.22$ & $0.00043^{*}$ \\
\hline
\end{tabular}

*Statistically significant differences (Student's $t$ test, $P<0.05$ )

of the lower level of iron in cancer patients could be bleeding of the malformed tissue. However, it seems unlikely since extensive bleeding should also decrease ferritin and the concentration of ferritin in carcinoma patients was even higher although, non-significantly than in the control group (Table 1).

Ferritin is an iron sequestering protein whose synthesis and concentration is governed by the labile iron pool. An increase of the intracellular iron pool leads to stimulation of ferritin synthesis [13]. To explain why the concentration of ferritin did not change according to the general trend in iron metabolism we decided to analyze the "labile iron pool". The analysis was performed in human lymphocytes. The LIP represents this part of intracellular iron which is in dynamic equilibrium with other intracellular iron forms and is bound to cytosolic low affinity ligands that have not yet been identified. This iron form is catalytically active and participates in reactions involved in the production of harmful reactive oxygen species (Fenton reaction) [1]. eters. Such huge individual differences in ferritin concentration were also reported by others [3]. Since this is the first in vivo study of LIP in human lymphocytes it is difficult to compare ours value with literature data. However, the concentration found is comparable to the levels estimated for different types of mammalian cultured cells [8]. We postulate that the higher concentration of LIP in the lymphocytes of patients with carcinoma may explain the distinctive behaviour of ferritin observed in our report, i.e. the high level of the intracellular LIP may cause an increase in ferritin synthesis and determine high plasma concentration of ferritin. Since the labile iron pool can influence production of reactive oxygen species our results support a recent observation that oxidative stress may be responsible for impaired lymphocyte function in cancer patients [5].

Usually, in malignant diseases plasma iron level falls due to cytokines activity [1]. Our observations are in favour of the interesting hypothesis, which suggests that the observed 
changes lead to restriction of iron availability for tumour cells to slow its growth [14]. However, a larger study group must be analyzed to detect statistically relationships, and the tendencies found in our results should be confirmed in the case of other types of cancer.

\section{R E F E R E N C E S}

1. Halliwell, B.M. \& Gutterige, J.M.C. (1999) Free Radicals in Biology and Medicine; 3rd edn. Oxford University Press Inc., New York.

2. Stevens, G., Graubard, B.I., Micozzi, M.S., Neriishi, K. \& Blumberg, B.S. (1994) Moderate elevation of body iron level and increased risk of cancer occurrence and death. Int. J. Cancer 56, 364-369.

3. Nelson, R.L., Davis, F.G., Sutter, E., Sobin, L.H., Kikendall, J.W. \& Bowen, P. (1994) Body iron stores and risk of colonic neoplasia. $J$. Natl. Cancer Inst. 86, 455-460.

4. Bird, C.L., Witte, J.S., Swendseid, M.E., Shikany, J.M., Hunt, I.F., Frankl, H.D., Lee, E.R, Longnecker, M.P. \& Haile, R.W. (1996) Plasma ferritin, iron intake, and the risk of colorectal polyps. Am. J. Epidemiol. 144, $34-41$.

5. Schmielau, J. \& Finn, O.J. (2001) Activated granulocytes and granulocyte-derived hydrogen peroxide are the underlying mechanisms of suppression of T-cell function in advanced cancer patients. Cancer Res. 61, 4756-4760.

6. Gackowski, D., Ciecierski, M., Jawien, A. \& Olinski, R. (2001) Background level of 8-oxo-2'-deoxyguanosine in lymphocyte DNA does not correlate with concentration of antioxidant vitamins in blood plasma. Acta Biochim. Polon. 48, 535-539.

7. Epsztejn, S., Kakhlon, O., Glickstein, H., Breuer, W. \& Cabantchik, I. (1997) Fluorescence analysis of the labile iron pool of mammalian cells. Anal. Biochem. 248, 31-40.

8. Lipinski, P., Drapier, J.-C., Oliveira, L., Retmanska, H., Sochanowicz, B. \& Kruszewski, M. (2000) Intracellular iron status as a hallmark of mammalian cell susceptibility to oxidative stress: A study of L5178Y mouse lymphoma cell lines differentially sensitive to $\mathrm{H}_{2} \mathrm{O}_{2}$. Blood 95, 2960-2966.

9. Stevens, R.G., Jones, D.Y., Micozzi, M.S. \& Taylor, P.R. (1998) Body iron stores and the risk of cancer. N. Engl. J. Med. 319, 1047-1052.

10. Knekt, P., Reunanen, A., Takkunen, H., Aromaa, A., Heliovaara, M. \& Hakulinen, T. (1994) Body iron stores and risk of cancer. Int. J. Cancer 56, 379-382.

11. Bendich, A. \& Cohen, M. (1990) Ascorbic acid safety: Analysis of factors affecting iron absorption. Toxicol. Lett. 51, 189-201.

12.Hallberg, L., Hulten, L. \& Gramatkovski, E. (1997) Iron absorption from the whole diet in men: How effective is the regulation of iron absorption? Am. J. Clin. Nutr. 66, 347-356.

13. Ponka, P. (2000) Iron metabolism: Physiology and pathophysiology. J. Trace Elem. Exp. Med. 13, 73-93.

14. Weinberg, E.D. (1996) The role of iron in cancer. Eur. J. Cancer Prevent. 5, 19-36. 